\title{
ZUR HIERARCHISIERUNG DER WISSENSCHAFTLICHEN ASSERTIONEN MIT METATEXTUELLEN ÄUßERUNGEN
}

\author{
DANUTA OLSZEWSKA \\ University Gdańsk
}

\begin{abstract}
The article presents the ways of ranking assertions or sequences of assercions in a scientific text, with the use of metatext explications. This ranking results from introducing into the text meta-phrases which explicitly or implicitly expresses the author's speech act. Speech acts signal on the one hand the assertions which are especially important, in opposition to the important ones, on the other, second-rate assertions opposing the primary ones.

The article aims to present the analysis of the text creating function of hierarchical metaphrases and the ways of realizing them.
\end{abstract}

\section{Einleitende Bemerkungen}

Wissenschaftliche Kommunikation zeichnet sich durch eine Vielfalt der Existenzformen aus. Diese Vielfalt resultiert einerseits aus der fachlich-inhaltlichen Differenziertheit des vermittelten Wissens, deren Ergebnis eine kaum überschaubare Zahl von wissenschaftlichen Fachbereichen ist, andererseits aus der Art des Sprachverhaltens, das zwei grundlegende Erscheinungsformen umfaßt, und zwar eine mündliche und eine schriftliche Kommunikation. Resultate der mündlichen Kommunikation wie z.B. Vorlesung, Vortrag oder Diskussion gelten als Diskurse, Produkte der schriftlichen Kommunikation dagegen sind Texte. Neben der grundsätzlichen Gemeinsamkeit zwischen Diskurs und Text: beide dienen zum Transfer wissenschaftlicher Erkenntnisse, besteht zwischen ihnen ein wesentlicher Unterschied im Entstehungszusammenhang. Während beim Diskurs, der in einer Sprechsituation entsteht, die Interaktanten einen zeitlichen und räumlichen Kontakt haben, so daß die Produktion und Rezeption zusammenfallen, vollzieht sich die Interaktion mittels der Texte in einer Schreibsituation, bei der Produktion und Rezeption getrennt sind. Die unterschiedliche Entstehungssituation, d.h. die Situationsgebundenheit bei Diskursen und die Situationsentbindung bei Texten betonen u.a. Ehlich (1983), BeckerMrotzek (1994), Graefen (1997) und Hoffmann (1997). 
Ein wissenschaftlicher Text stellt ein komplexes Gebilde dar, in dem verschiedene funktionale Ebenen miteinander verflochten sind. Die am meisten ins Auge springende Ebene ist die der Propositionen, d.h. der vermittelten Inhalte. Im Hinblick auf den Zweck der wissenschaftlichen Texte, der sich mit der Überlieferung des neuen Wissens verbindet, gilt diese Ebene als primäre Ebene wissenschaftlicher Texte. Die inhaltlichen Strukturen und die mit ihnen zusammenhängende thematische Entfaltung kongruieren mit einer Ebene, die im Hinblick auf den Produktionsprozeß eines wissenschaftlichen Textes, seinen Werdegang, als eine erste und in diesem Sinne primäre Ebene aufgefaßt werden könnte. Gemeint ist hier das sprachliche Handeln des Autors beim Textaufbau, dessen Resultat eine Vertextung und Verkettung von Sprachhandlungen sind. Diese Handlungsstrukturen, die mit der Planung und Gestaltung des Textes verbunden sind, bilden eine metatextuelle Ebene wissenschaftlicher Texte. Auf dieser Ebene informiert der Autor den Leser explizit darüber, was er tut, was er bereits getan hat bzw. was er später tun wird. So entstehen sparsam oder reichlich dosierte Kommentierungen und Erläuterungen zum Text selbst, die syntaktisch sehr oft in Form eines vollständigen Satzes, manchmal mehrerer Sätze, formuliert werden und die gleichzeitig Repräsentationen und Manifestationen det Text- und der Sprachhandlungen sind. In der Fachliteratur werden sie meist unter dem Begriff "metakommunikative Äußerungen" zusammengefaßt (z.B. Techtmeier 1984; Timm 1987; Gläser 1990; Busch-Lauer 1991). Graefen nennt sie Textkommentierungen und betont ihre textorganisierende Funktion: „Sie dienen dem Autor dazu, den Leser über die Organisation des Textes explizit zu informieren, sowohl im Sinne einer Orientierungshilfe für den Leser als auch z.B. zum Zweck einer Legitimation" (Graefen 1997:160). Im folgenden nennen wir die behandelten Formulierungen metatextuelle Äußerungen oder kurz: Meta-Phrasen und wollen ausgewählte Typen von ihnen näher charakterisieren. Im Mittelpunkt der Analyse stehen Meta-Phrasen, die in einem wissenschaftlichen Text dazu dienen, Assertionen zu hierarchisieren. Den Begriff Assertion verwenden wir als Oberbegriff für Feststellungen, Behauptungen, Mitteilungen, kurz gesagt für elementare Äußerungseinheiten mit dem semantischen Merkmal „sagen". Grundlage der folgenden Übersicht bildet die Auswertung von 12 Monographien aus dem linguistischen Fachgebiet.

\section{Zur Analyse der hierarchisierenden Meta-Phrasen}

Ein wissenschaftlicher Text stellt eine Assertionskette dar, deren elementare Einheiten einfache, d.h. in Form eines Satzes formulierte Assertionen sind. Im Grunde genommen ist jeder Satz im Deklarativmodus im wissenschaftlichen Text eine Assertion, denn in jedem Satz vermittelt der Autor eine neue Information. Dennoch gibt es Stellen im Text, in welchen ein verb dicendi diese Auto- 
renhandlung in einem Hauptsatz explizit kennzeichnet, bevor eine Assertion in Form eines daß-Satzes angeschlossen wird, wie z.B. im Satz: Dabei ist zu betonen, daß p. Die einleitende Phrase Dabei ist zu betonen, daß ,ragt“ aus dem Text „heraus“ als ein Äußerungsteil, der auf den Kommunikationsprozeß, d.h. auf den Vollzug der Handlung des Sagens vom Autor explizit Bezug nimmt. Dieser Art explizite Kommunikationsbezüge sind für wissenschaftliche Texte äußerst charakteristisch und werden von den Autoren an verschiedenen Textstellen benutzt, wenn sie etwas „Spezielles“ zu sagen haben, worauf die Aufmerksamkeit des Lesers fokussiert werden soll. In der Fülle der aneinandergereihten und neues Wissen enthaltenen Assertionsketten legt der Autor eine gewisse Ordnung fest und signalisiert, daß er die Assertionen nicht gleichrangig behandelt. Daher markiert er, welche Information von seinem Standpunkt aus im gegebenen Zusammenhang z.B. wichtiger als eine andere ist oder welche Information vordergründig ist und zum „roten Faden“ des Gesamtinhalts gehört und welche als Hintergrund gelten soll. Auf diese Weise hierarchisiert der Autor die Assertionen und die mit ihnen verbundenen Inhalte, anders: er strukturiert sie unter Relevanzaspekten. Die Hierarchisierung ermöglicht dem Autor, spezifische Inhalte des Gesagten in den Fokus des Lesers zu bringen. Die Fokussierung als Methode, die Aufmerksamkeit des Lesers auf spezifische Informationsgehalte zu lenken, löst Veränderungen im Bereich des Wissens aus und trägt zur entsprechenden Verarbeitung dieses Wissens bei (Hoffmann 1997:232).

Was im Text als spezifische Assertion markiert und damit in den Fokus des Lesers gebracht werden soll, ist die individuelle Leistung des Autors. Auf eine subjektive und arbiträre Weise entscheidet er, welche Assertionen als spezielle Gehalte sich in der linearen Abfolge auszeichnen sollen. Das gesammelte Material weist darauf hin, daß die Hierarchisierung vor allem auf zwei Ebenen vorgenommen wird:

- Assertion: relevant vs. besonders relevant

- Assertion: vordergründig vs. hintergründig

Als spezielle Assertionen, die im Text durch Meta-Phrasen eingeleitet und damit herausgestellt werden, gelten: besonders relevante Assertionen und hintergründige Assertionen. Besonders relevante Assertionen stehen in der Hierarchie höher als die relevanten, die man als eine Norm betrachten kann. Hintergründige Assertionen dagegen stehen in der Hierarchie niedriger als die vordergründigen, die ebenfalls eine Norm bilden. Man kann also sagen: besonders relevante Assertionen und hintergründige Assertionen bilden zwei Extrempunkte einer Skala. Zwischen ihnen befinden sich Assertionen mit Inhalten, die unterschiedlichen Grad von Relevanz und Hintergrund haben und auf die ein Autor mehr oder weniger hinweisen will.

Besonders relevante Assertionen werden durch die Sprechhandlung des Hervorhebens signalisiert. Mit dem Vollzug dieser Handlung verbindet sich 
eine Reihe von Meta-Phrasen, wie z.B. Hervorzuheben ist dabei, daß $p$ oder An dieser Stelle muß betont werden, daß $p$.

Assertionen mit hintergründigen Inhalten werden durch die Sprechhandlung des Hinzufügens markiert. Der Vollzug dieser Handlung führt ebenfalls zu verschiedenen Meta-Phrasen, wie z.B. An dieser Stelle sei hinzugefügt, daß $p$ oder Nur am Rande soll hier angemerkt werden, daß p. Die dazwischen liegenden Inhalte befinden sich entweder in der Nähe der besonders relevanten Assertionen und sind an die Handlungen des Hervorhebens, des Hinweisens, des Aufmerksammachens gebunden oder in der Nähe der (nur) hintergründigen Assertionen und sind Resultate der Handlungen des Hinzufügens, des Ergänzens, des Anmerkens. Als zusätzliche Ebene, auf der eine Gewichtung der wissenschaftlichen Assertionen vorgenommen wird, kann man die Ebene: neutral vs interessant betrachten, die dazu dienen kann, interessante Informationen bei der Behandlung eines Problems zu signalisieren. Dies kommt z.B. in solchen Meta-Phrasen zum Ausdruck wie: Interessant ist dabei, daß p oder Bemerkenswert ist dabei, daß $p$.

Insgesamt verbinden sich mit den genannten Handlungen: des Hervorhebens, des Hinweisens, des Aufmerksammachens, des Hinzufügens, des Anmerkens und des Ergänzens sowie mit ihren metatextuellen Repräsentationen Assertionen besonderer Art, die wir unter zwei Hauptarten von Assertionen zusammenfassen wollen: Hervorhebungen und Hinzufügungen.

Der propositionale Gehalt der als Beispiele angeführten Meta-Phrasen hat eine offene Struktur und kann alles erfassen, was nur hervorhebbar bzw. hinzufügbar ist. Im Falle des Hervorhebbaren geht es um allgemeine oder spezifische Informationen, die dem Autor aus Kommunikationszwecken als wichtig und notwendig zum Verstehen des erörterten Problems erscheinen. Es können autorbezogene Propositionen sein, wie z.B. wichtige Bemerkungen zum Standpunkt des Autors oder sog. weltbezogene Propositionen, wie z.B. Bemerkungen zu den allgemeinen Tendenzen, zum Regulären und Irregulären im untersuchten Bereich, zu den fließenden Grenzen, zu den Gemeinsamkeiten und Unterschieden, kurz gesagt, alle Propositionen, die mit dem dargelegten Problem im unmittelbaren Zusammenhang stehen und zum inhaltlichen Kern gehören. Beim Hinzufügen kommen eher Nebenbemerkungen in Frage. Sie können einen allgemeinen oder spezifischen Charakter haben. Sie können ebenfalls autorbezogene Propositionen, z.B. Nebenbemerkungen zur Vorgehensweise des Autors oder weltbezogene Propositionen sein, z.B. Nebenbemerkungen zum erfaßten Korpus, zur Frequenz der untersuchten Belege, zu den Ausnahmen und Abweichungen von der Norm, kurz gesagt, alle Propositionen, die mit dem behandelten Problem mittelbar im Zusammenhang stehen, zusätzliche Informationen zum inhaltlichen Kern bilden und die keinen unmittelbaren Einfluß auf das Verstehen des Problems haben. Der Autor fühlt sich jedoch verpflichtet, sie zu übermitteln, er kann sie aber im Rahmen seiner Arbeit nicht weiter entwickeln. 
Auf die Frage, an welchen Textstellen die genannten Handlungen vollzogen werden, d.h. gleichzeitig in welche Textstrukturen die Hervorhebungen und Hinzufügungen eingebettet werden, ist zunächst festzustellen, daß sie Elemente der Mikrostrukturen sind, d.h. auf der Ebene eines Absatzes Anwendung finden. Sie können:

- einen Absatz eröffnen und als initiale Phrase mehrere Assertionen einleiten,

- absatzintern stehen und im Rahmen der Textfortführung mehrere Assertionen umfassen,

- einen Absatz abschließen, abrunden und eine Assertion einleiten, die das Thema „stoppt”.

Metatextuell wirken die hierarchisierenden Äußerungen sowohl links- als auch rechtsseitig ein. Ihre linksseitige Einwirkung besteht darin, daß die ihnen angeschlossene Assertion die obigen Gehalte des Textraums erweitert, ergänzt. Gleichzeitig „kündigen" diese Phrasen ein spezielles Informationsgehalt „an“. Im Falle des Hervorhebens ist das eine besonders relevante, mit dem inhaltlichen Kern des Gesamttextes unmittelbar verbundene Information; im Falle des Hinzufügens ist das eine (nur) hintergründige Information, die in einem mittelbaren Zusammenhang mit dem behandelten Thema steht und im Rahmen der jeweiligen Arbeit vom Autor nicht näher betrachtet werden kann. Das bedeutet natürlich nicht, daß eine hintergründige Information als wenig wichtig bzw. gar unwichtig gilt. Sie ist zwar eine zusätzliche, ergänzende, jedoch wichtige Information, die dem vorhandenen Wissen nur lokal oder global hinzugefügt wird, aber nicht mehr entwickelt werden kann. Davon zeugt z.B. die Meta-Phrase: Ergänzend soll man hier betonen, daß $p$, in der zwei von uns genannten Extrempunkte der hierarchisierenden Ausdrucksmittel zusammentreffen: das Hervorheben und das Ergänzen/Hinzufügen. Diese „hybride“ Handlungskombination vermittelt dem Leser folgende Informationen: Die linksseitige Information ist für beide Handlungen gleich und kann verstanden werden: Der Autor hat zu dem gerade behandelten Problem noch nicht alles gesagt und wird jetzt das Gesagte erweitern, ergänzen. Was ihre rechtsseitige Einwirkung auf die unmittelbare Textumgebung anbetrifft, so unterscheiden sich die beiden Sprechhandlungen durch ihr Informationsgewicht voneinander. Der Ausdruck betonen, der hier als expliziter Träger der Handlung auftritt und den man als Meta-Prädikat bezeichnen kann, vermittelt dem Leser die Information: Der Autor sagt jetzt etwas, was von seinem Standpunkt aus im gegebenen Zusammenhang besonders relevant ist und zum inhaltlichen Kern des Gesamttextes gehört. Der Ausdruck ergänzend dagegen, der in dieser Phrase für die Handlung des Hinzufügens steht, vermittelt dem Leser die Information: Der Autor sagt jetzt etwas, was zwar mit dem gerade erläuterten Problem zusammenhängt, was aber vom Autor als zusätzliche Information betrachtet wird, die keinen unmittelbaren 
Einfluß auf das Verstehen des Problems hat und die an der jeweiligen Textstelle nicht entwickelt wird. Der Autor fühlt sich aber verpflichtet, auf diese Information zumindest kurz hinzuweisen. In solchen Fällen leiten dieser Art metatextuelle Phrasen Assertionen ein, die ein Thema abschließen, abrunden bzw. „stoppen“, damit der Text seinen logischen Aufbau bewahrt und den „roten Faden" nicht verliert.

Zum Einwirkungsbereich der hierarchisierenden Meta-Phrasen ist folgendes zu bemerken: Werden sie als initiale Subthematisierungen oder bei Themafortführung verwendet, so können sie einen ganzen Absatz unterschiedlichen Umfangs bilden. Sind sie gegen Ende eines Absatzes positioniert und dem Themastoppen dienen sollen, dann kann das von ihnen eröffnete spezielle Informationsfeld nur aus einem Satz bestehen. In beiden Fällen haben wir die hervorzuhebenden oder hinzuzufügenden Assertionen als $p$ kenntlich gemacht.

Insgesamt zählen wir zu den hierarchisierenden Meta-Phrasen in erster Linie diejenigen, die sich um folgende typische handlungsbezogene Ausdrücke gruppieren:

betonen, hervorheben, hinweisen, aufmerksam machen, ergänzen, hinzufügen, erwähnen, anmerken, vermerken, bemerken, herausstellen, einschränkend sagen

Durch die höchste Frequenz zeichnen sich in unserem Textkorpus die drei ersten explizit handlungswiedergebenden Verben aus, die im Hinblick auf die Funktionen und den Gebrauch vergleichbar sind. Als initiale Subthematisierungen, die einen mehrere Assertionen umfassenden Absatz bilden, treten sie in Verbindung mit temporalen Angaben auf, z.B.:

Am Anfang ist zu betonen, daß ...

Zunächst muß hervorgehoben werden, daß ..

Was ... anbelangt, so ist zunächst darauf hinzuweisen, daß ...

Um die Relationen zwischen ... zu bestimmen, muß hier zuerst auf die Begriffe hingewiesen werden, die ...

Bei einer absatzinternen Stellung, die auf ihre themafortführende Funktion hindeutet, verknüpfen sie sich mit lokalen paradeiktischen Mitteln wie: hier, an dieser Stelle, dabei, im Zusammenhang, mit deren Hilfe Bezug auf die unmittelbare Textumgebung genommen wird. Typisch sind auch „fortsetzungsstiftende" Partikeln wie: noch, darüber hinaus, schließlich sowie Partikeln mit ,polemisierendem" Charakter wie: allerdings, jedoch, die mit der lokalen Deixis kombiniert werden können. Beispiele:

Es ist dabei zu betonen, daß

Betont werden muß dabei allerdings, daß ...

Andererseits sei hier betont, daß ...

Darüber hinaus gilt es an dieser Stelle zu betonen, daß ...

Schließlich soll hier noch auf die Fälle hingewiesen werden, wo ... 
Davon, daß es sich bei diesen Meta-Phrasen um die Markierung der besonders relevanten Assertionen handelt, zeugen die beteiligten Ausdrücke, die ihre besondere Relevanz zusätzlich kennzeichnen. Zu ihnen gehören: iterative Ausdrücke wie: noch einmal, nochmals, wiederholt, intensivierende Ausdrücke wie: deutlich, mit aller Deutlichkeit, mit Nachdruck sowie die Ausdrücke notwendig und wichtig, die als Teile der Prädikativsätze auftreten. Beispiele:

Es sei hier noch einmal mit $x$ betont, daß ...

Dabei müssen wir wiederholt betonen, daß ...

So ist hier mit aller Deutlichkeit zu betonen, daß ...

Aus diesem Grunde ist mit Nachdruck darauf hinzuweisen, daß ...

Da dies für ... der ... wichtig ist, sei noch einmal darauf hingewiesen, daß ...

..., daher ist es notwendig, die Tatsache hervorzuheben, daß ...

In der themaabschließenden (,themastoppenden ${ }^{65}$ ) Funktion verknüpfen sich die genannten Meta-Prädikate zur Markierung besonders relevanter Gehalte mit zweierlei Ausdrucksformen. Die erste Kategorie bilden modale oder temporale Angaben wie: ergänzend, abschließend, zum Abschluß. Zur zweiten Kategorie gehören vollständige Sätze, die die Unterlassung einer Handlung signalisieren. In diesen Fällen enthalten die metatextuellen Phrasen oft eine einschränkende Gradpartikel, und zwar nur oder lediglich z.B.:

Ergänzend möchte ich betonen, daß...

Abschließend wollen wir betonen, daß...

Ohne auf eine weitere Auseinandersetzung einzugehen, möchte ich betonen, daß...

Ohne daß wir an dieser Stelle das Problem diskutieren möchten, wollen wir auf den Umstand hinweisen, daß...

Da dieses Thema im Zusammenhang mit... wieder aufgegriffen wird, soll diese Frage nicht fortgesetzt werden. Es sei aber nur darauf hingewiesen, daß...

Die Möglichkeit der... können wir hier nicht weiter diskutieren; es soll an dieser Stelle lediglich darauf hingewiesen werden, daß...

Die anderen handlungswiedergebenden Verben und verbalen Fügungen aus der Gruppe, die zur Markierung besonders relevanter Assertionen dienen, weisen eine deutlich niedrigere Frequenz auf. Die mit ihnen ermittelten Beispiele sind:

Abschließend möchte ich herausstellen, daß...

Zum Schluß sollte noch darauf aufmerksam gemacht werden, daß...

Auf der zweiten Ebene der Hierarchisierung, auf der hintergründige Assertionen von den vordergründigen unterschieden werden, spielen die explizit handlungsbezeichnenden Verben hinzufügen, anmerken und erwähnen die größte Rolle, d.h. sie gehören zu den hochfrequenten Meta-Prädikaten. Sie konstituieren metatextuelle Phrasen, mit deren Hilfe der Autor zu den vorhandenen essentiellen Inhalten zusätzliches repräsentatives Wissen hinzufügen will. Innerhalb eines 
Abschnitts können sie gebraucht werden als initiale Meta-Phrasen, die mehrere Assertionen einleiten. Sie eröffnen ein neues spezielles Informationsfeld, das einen Absatz am Ende eines Abschnitts konstituiert, z.B.:

Eine weitere wichtige Frage, die dabei erwähnt werden muß, betrifft ...

Das Problem der ... soll hier nur kurz erwähnt werden, da es mit den hier ... nur mittelbar im Zusammenhang steht.

Zum Abschluß dieses Überblicks sei noch eine weitere Richtung erwähnt, die ...

Abschließend soll nur noch die letzte Klasse erwähnt werden, und zwar ...

Zur Frage der ... sollen hier noch drei Problemkreise angemerkt, ohne die aufgeworfene Frage hier gültig beantworten zu wollen. Zunächst:...

oder als absatzfinale Meta-Phrasen, die dazu dienen, ein Thema abzuschließen. Sie eröffnen ein neues spezielles Informationsfeld, das gegen Ende eines Absatzes in Form eines daß-Satzes angeschlossen wird. Im Skopus der sprechaktbezeichnenden Meta-Prädikate befinden sich: die auf den Nahbereich orientierenden textdeiktischen Ausdrücke hier oder dabei sowie die auf den ergänzenden Charakter dieser Assertionen verweisenden Einheiten wie: noch, nur kurz, einschränkend, z.B.:

Zu der hier dargestellten ... müssen wir einschränkend erwähnen, daß ...

Hier muß noch erwähnt werden, daß ...

Angemerkt sei noch, daß ...

$Z u$ der Konzeption von $x$ sei kritisch angemerkt, daß ...

Nur am Rande soll/muß hier angemerkt werden, daß ..

Es ist dabei anzumerken, daß ...

In einer ähnlichen Form und Funktion werden die übrigen handlungsbezeichnenden Ausdrücke aus der Gruppe verwendet, von welchen das Meta-Prädikat hinzufügen in unserem Korpus die höchste Frequenz hat. In Verbindung mit solchen Ausdrücken wie hier, dabei, ergänzend, allerdings, gleichzeitig, aber dienen sie zur Einbettung der ergänzenden Assertionen, die den inhaltlichen Kern des Gesamttextes um zusätzliche Gehalte erweitern. Die metatextuellen Phrasen mit ihnen leiten nur einfache, d.h. nur einen Satz umfassende Assertionen ein. Beispiele:

Es ist (hier) ergänzend hinzuzufügen, daß ...

Um die Terminologie zu ergänzen, sei hinzugefügt, daß ...

Hier muß man/müßte man hinzufügen, daß ...

Hinzuzufügen ist, daß ...

Es muß gleichzeitig aber vermerkt werden, daß ...

Es muß/soll ergänzend gesagt werden, daß ...

Assertionen mit speziellen Gehalten markiert der Autor nicht nur durch explizit handlungsbezeichnende Verben des Hervorhebens und Hinzufügens. Die Auf- 
merksamkeit des Lesers wird auf besonders relevante oder hintergründige Gehalte auch mit Hilfe der metatextuellen Phrasen fokussiert, die keine Handlungsverben enthalten, sondern Verben, die mental-visuelle Zustände oder Operationen bezeichnen und die man unter dem Oberbegriff des Beachtens von (noch) etwas zusammenfassen kann. Sie dienen dem Autor vor allem dazu, (Neben)bemerkungen zu machen. Es handelt sich um folgende Verben, die als Meta-Prädikate in wissenschaftlichen Texten benutzt werden:

beachten, bemerken, berücksichtigen, erinnern, vergessen, übersehen u.ä.

Typische Meta-Phrasen mit den genannten Verben enthalten eine sein $+z u$ + Infinitiv-Konstruktion, die hier Ausdruck für eine Notwendigkeit ist. Die Verben übersehen und vergessen treten in negierten Formen auf. Die beteiligten Ausdrücke, die eine lokale absatzinterne Stellung dieser spezielle Gehalte einleitenden Phrasen indizieren, sind: dabei, an dieser Stelle, in diesem Zusammenhang u.ä. Beispiele:

Dabei ist $z$ u beachten, daß ...

$\mathrm{Zu}$ beachten ist in diesem Zusammenhang, daß ...

In ... Hinsicht ist zu berücksichtigen, daß ..

$\mathrm{Zu}$ berücksichtigen ist dabei/Zu bemerken ist dabei, daß ...

Es ist jedoch zu bemerken/Es bleibt noch zu bemerken, daß ...

Es ist (hier) daran zu erinnern, daß ..

Nicht zu vergessen ist dabei, daß ...

Nicht zu übersehen ist in diesem Zusammenhang, daß ...

Eine Notwendigkeit wird auch mit Hilfe des adhortativen Konjunktivs ausgedrückt; allerdings klingt er nicht so kategorisch, wie die infinitivischen Konstruktionen (vgl. Engel 1999:608). Der Autor hält es für erwünscht, diese (Neben)bemerkung zu machen, vgl.:

An dieser Stelle sei daran erinnert, daß..

Es ist daran zu erinnern, daß...

Als weitere funktionsähnliche Alternativen stehen dem Autor die Modalverben müssen und sollen zur Verfügung:

Man soll/sollte dabei beachten/bemerken, daß ...

Man muß jedoch einschränkend bemerken, daß ...

Dazu müßte man folgendes bemerken:

Nicht vergessen sollte man, daß ...

Man sollte jedoch nicht übersehen, daß ...

Schließlich kann man zum Ausdruck des Erwünschten das adjektivische Halbsuffix -wert ausnutzen, wenn man auf etwas Bedeutendes/Interessantes hinweisen will, z.B.: 
Bemerkenswert/Beachtenswert ist dabei, daß ...

In den Tiefenstrukturen dieser Phrasen steckt ein direktiver Akt, der Ausdruck für ein interaktionelles Handeln des Autors ist. Die in ihnen enthaltene Textrolle „Leser" kommt in den Paraphrasen zum Ausdruck:

Beachten/Berücksichtigen/Bemerken Sie bitte dabei, daß ...

Übersehen Sie bitte nicht/Vergessen Sie bitte nicht, daß ...

Auch die Textrolle „Autor“ ist in diesen Strukturen enthalten:

Ich möchte Sie an dieser Stelle daran erinnern, daß ...

Ich möchte dabei auch bemerken, daß ...

Ich möchte dabei noch die Tatsache berücksichtigen, daß ...

Aus den zahlreichen Beispielen zu den hierarchisierenden Meta-Phrasen ist ersichtlich, daß bei der Formulierung spezieller Assertionen, d.h. der Assertionen mit besonders relevanten Gehalten einerseits und mit hintergründigen, ergänzenden Gehalten andererseits verschiedene sprachliche Indikatoren Anwendung finden. Unter ihnen sind Meta-Phrasen mit expliziten Handlungsprädikaten sehr charakteristisch. Die Realisierungsformen der hierarchisierenden Meta-Phrasen sind für beide Arten der speziellen Assertionen, d.h. für Hervorhebungen und Hinzufügungen vergleichbar und lassen sich durch folgende Merkmale charakterisieren: „,agensorientiert”, „deagentiviert”, „modalisiert”, „handlungsbezogen” (das Meta-Prädikat ist Handlungsverb), „nicht handlungsbezogen" (das Meta-Prädikat ist nicht Handlungsverb). Aus der Kombination dieser Merkmale ergeben sich folgende Hauptmuster für MetaPhrasen, die spezielle Assertionen einleiten:

- Merkmalkombination: „agensorientiert” + „modalisiert” + „handlungsbezogen”

- Realisierungsform: Adverbial + ich/wir + möchte/n/will/wollen + Handlungsverb. „Adverbial" wird als Adverbphrase oder Präpositionalphrase realisiert. Darunter sind temporale, lokale oder modale Ausdrücke zu verstehen.

- Handlungsverben mit der semantischen Komponente „sagen”: betonen, hervorheben, hinweisen, aufmerksam machen, ergänzen, hinzufügen, erwähnen, anmerken, vermerken, herausstellen, einschränkend sagen

- Merkmalkombination: „deagentiviert” + „modalisiert” + „handlungsbezogen”.

- Realisierungsform: Adverbial + Passivkonstruktion mit müssen/sollen + Handlungsverb

- Realisierungsform: Adverbial + man-Konstruktion mit müssen/sollen + Handlungsverb

- Realisierungsform: Adverbial + Konjunktiv I + Handlungsverb (Verben wie oben)

- Merkmalkombination: „deagentiviert” + „modalisiert” + ,handlungsbezogen” oder „nicht handlungsbezogen" 


\section{Realisierungsform:}

\section{Adverbial + Passivparaphrase sein $+z$ Wes gilt $+($ Nicht $)$ Handlungsverb}

- Handlungsverben wie oben,

- Nichthandlungsverben mit der semantischen Komponente „beachten”: bemerken, beachten, berücksichtigen, erinnern, vergessen, übersehen. Realisierungsform: einleitender Prädikativsatz + Infinitivkonstruktion mit Handlungsverb. Es handelt sich um Prädikativsätze mit Bewertungsfunktion, z.B. Es ist wichtig/notwendig/legitim/angemessen u.ä. Handlungsverben wie oben,

- Merkmalkombination: „deagentiviert” + „modalisiert” + „nicht handlungsbezogen". Realisierungsform: (Adverbial) + Verb sein + Adjektiv mit -wert,

- Nichthandlungsverben mit der semantischen Komponente „beachten”: bemerken, beachten

Aus der Analyse der Meta-Phrasen und aus den angegebenen Mustern geht deutlich hervor, daß für diesen Bereich modalisierte Formeln als sog. verdeckte Performativa fast eine Regel bilden. Dabei ist weiterhin zu bemerken, daß sowohl die Assertionen mit besonders relevanten Gehalten als auch die mit hintergründigen, ergänzenden Gehalten am häufigsten durch Meta-Phrasen mit der Modalität einer sicheren Gültigkeit und einer objektiven Notwendigkeit eingeleitet werden. Unabhängig davon, ob der Autor besonders relevante Gehalte hervorhebt oder zusätzliche, hintergründige Gehalte zum vorhandenen Wissen nur hinzufügen will, tut er das mit der Einstellung: Ich halte diese spezielle Information für notwendig im gegebenen Zusammenhang. Die besonders relevanten Gehalte muß ich hervorheben, weil sie zum inhaltlichen Kern gehören; die hintergründigen Informationen sind ebenfalls wichtig, weil sie das Gesamtbild einer Untersuchung differenziert darstellen und vervollständigen. Auf diese Weise eingeleitete Assertionen ergeben sich als eine zwangsläufige Folge gewisser unabhängig vom Autor und Leser existierender objektiver Umstände. Über die mit müssen modalisierten Sprechakte in den wissenschaftlichen Texten sagt Panther folgendes: Sie dienen dazu, ,die Wichtigkeit der im propositionalen Teil ausgedrückten Informationen zu signalisieren. Der Wissenschaftler will sich nicht dem Vorwurf aussetzen, wichtige Informationen verschwiegen zu haben, deswegen fühlt er sich verpflichtet, diese Information zu geben " (Panther 1981:254). Die Funktion der Modalität „objektive Notwendigkeit” besteht also beim Vollzug des Hervorhebens und Hinzufügens darin, diese Handlungen als legitime zu kennzeichnen. Die legitim vollzogenen Sprechhandlungen dienen wiederum dazu, wissenschaftliche Assertionen zu hierarchisieren und aus der Fülle der Informationen rezeptionsrelevante Inhalte herauszustellen.

\section{Zusammenfassung}

Ein wissenschaftlicher Text hat eine komplexe Organisationsstruktur, in der sich viele sprachliche Handlungen manifestieren. Ein großer Teil von ihnen erscheint auf der Textoberfläche und bildet die äußere, metatextuelle Ebene des 
Textes, auf der ein Autor über seine Handlungen informiert. Das Resultat sind metatextuelle Äußerungen, denen der Leser sowohl auf der Makro- wie auch Mikroebene eines Textes begegnet. Die im folgenden Beitrag behandelten metatextuellen Äußerungen sind Elemente der Mikroebene, d.h. der Ebene des Absatzes, der aus den elementarsten Äußerungseinheiten besteht, und zwar aus den Assertionen. Darunter verstehen wir Äußerungen mit der semantischen Komponente „sagen”. Ein wissenschaftlicher Text stellt eine Verkettung von assertorischen $\ddot{A} u ß e r u n g e n$ dar. Unter ihnen haben Hervorhebungen und Hinzufügungen einen besonderen Status und werden als spezielle Assertionen mit Hilfe metatextueller Äußerungen herausgestellt. Ihre Herausstellung besteht darin, daß die genannten Assertionstypen durch metatextuelle Phrasen eingeleitet werden, mit welchen der Bezug auf den Autor und seine Handlung genommen wird. In dieser Herausstellung sehen wir ein Verfahren zur Hierarchisierung wissenschaftlicher Assertionen. Die Hauptfunktion dieser metatextuellen Äußerungen besteht darin, den Leser auf besonders relevante Inhalte einerseits (bei Hervorhebungen) und nur hintergründige Nebenbemerkungen andererseits (bei Hinzufügungen) $\mathrm{zu}$ orientieren. Eine metatextuelle Äußerung ist meistens ein Hauptsatz, dem eine in Form eines daß-Satzes formulierte spezielle Assertion angeschlossen wird. Deshalb sprechen wir hier auch von einleitenden MetaPhrasen. In ihnen manifestieren sich viele Handlungen des Autors, die man zwei genannten Grundtypen zuordnen kann. Die Handlungen werden entweder explizit, d.h. mit Hilfe der auf der Oberfläche der Sätze erscheinenden sprechaktbezeichnenden Verben realisiert oder implizit mit anderen nicht sprechaktbezeichnenden Verben. Im ersten Falle sprechen wir von handlungsbezogenen, im zweiten von nicht handlungsbezogenen Meta-Phrasen. In jedem wissenschaftlichen Text lassen sich beide stilistische Typen der metatextuellen Äußerungen beobachten. Innerhalb beider Stile sind verschiedene syntaktische Varianten der metatextuellen Phrasen möglich, die sich zu einigen Hauptmustern einordnen lassen.

Zum Thema der metatextuellen Äußerungen in wissenschaftlichen Texten liegen bisher kaum linguistische Analysen vor. Die Thematik ist für alle wissenschaftlichen Schreibprozesse in allen Fächern außerordentlich relevant, insbesondere aus der Perspektive des Deutsch lernenden Ausländers. Ihre Kenntnis gibt den jungen ausländischen Germanisten Instrumente in die Hand, mit welchen sie ihre wissenschaftlichen Arbeiten besser planen und gestalten können.

\section{QUELLEN}

Bilut-Homplewicz, Zofia (1998): Zur Dialogtypologie in der Erzählung aus textlinguistischer Sicht. Rzeszów.

Czarnecki, Tomasz (1998): Aspektualität im Polnischen und Deutschen. Bedeutungen und Formen in einer konfrontativen Übersicht. Gdańsk.

Dahl, Johannes (1988): Die Abtönungspartikeln im Deutschen. Ausdrucksmittel für Sprechereinstellungen mit einem kontrastiven Teil deutsch-serbokroatisch. Heidelberg. 
Dębski, Antoni (1989): Studien zum Lexikon als Komponente einer deskriptiven Grammatik. Zu Lexikon-Einträgen für Verben auf der Grundlage der semantischen Valenztheorie. Krakow.

Fiedler, Sabine (1991): Fachtextlinguistische Untersuchungen zum Kommunikationsbereich der Pädagogik - dargestellt an relevanten Fachtextsorten im Englischen. Frankfurt/M.

Öhlschläger, Günther (1989): Zur Syntax und Semantik der Modalverben des Deutschen. Linguistische Arbeiten 144. Tübingen.

Prokop, Izabela (1995): Erotetische Sprechakte im Deutschen und im Polnischen anhand natürlicher Gespräche. Poznań.

Schank, Gerd; Schoenthal, Gisela (1976): Gesprochene Sprache. Eine Einführung in Forschungsansätze und Analysemethoden. Germanistische Arbeitshefte. Tübingen.

Schneider, Edgar W. (1988): Variabilität, Polysemie und Unschärfe der Wortbedeutung. Bd. 1: Theoretische und methodische Grundlagen. Linguistische Arbeiten 196. Tübingen.

Wawrzyniak, Zdzisław (1980): Einführung in die Textwissenschaft. Probleme der Textbildung im Deutschen. Warszawa.

Wiese, Ingrid (1984): Fachsprache der Medizin. Eine linguistische Analyse. Leipzig.

Wiktorowicz, Józef (1985): Semantische Analyse der Adjektive der Sinneswahrnehmung in der deutschen Gegenwartssprache. Warszawa.

\section{LITERATUR}

Becker-Mrotzek, Michael (1994): Schreiben als Handlung. In: Brünner Gisela/Graefen. Gabriele (Hg.), Texte und Diskurse. Opladen, 158-175.

Busch-Lauer, Ines-Andrea (1991): Englische Fachtexte in der pädagogischen Psychologie. Eine linguistische Analyse. Frankfurt/M.

Ehlich, Konrad (1983): Text und sprachliches Handeln. Die Entstehung von Texten aus dem Bedürfnis nach Überlieferung. In: Assmann, Aleida; Assmann, Jan; Hardmeier, Christof (Hg.), Schrift und Gedächtnis. Beiträge zur Archäologie der literarischen Kommunikation. München, 24-43.

Engel, Ulrich (1999): Deutsch-polnische kontrastive Grammatik. Unter Mitarbeit von Danuta Rytel-Kuc u.a. Bd. 1. Heidelberg.

Gläser, Rosemarie (1990): Fachtextsorten im Englischen. Tübingen.

Graefen, Gabriele (1997): Der Wissenschaftliche Artikel - Textart und Textorganisation. Frankfurt/M.

Hoffmann, Ludger (1997): Thematische Organisation von Text und Diskurs. In: Zifonun, Gisela; Hoffmann, Ludger; Strecker, Bruno et al. (Hg.), Grammatik der deutschen Sprache. Berlin.

Panther, Uwe (1981): Indirekte sprachliche Handlungen im wissenschaftlichen Diskurs. In: Bungarten, Theo (Hg.), Wissenschaftssprache. München, 231-260.

Techtmeier, Bärbel (1984): Das Gespräch. Funktionen, Normen und Strukturen. Berlin.

Timm, Chrstian (1987): Gibt es eine Fachsprache der Literaturwissenschaft? Fachtextlingusitische Untersuchungen an englischen Texten der Literaturgeschichtsschreibung. Frankfurt/M. 